\title{
Ultrasound-assisted synthesis of eight novel and highly functionalized 2-aminonitrile oxazoles via Ugi-3CR
}

Manuel A. Rentería-Gómez, ${ }^{\mathrm{a}}$ Ivette Morales-Salazar, ${ }^{\mathrm{b}}$ Natalia García-González, ${ }^{\mathrm{b}}$ Daniel Segura-Olvera, ${ }^{\mathrm{b}}$ Mayra Sánchez-Serratos, ${ }^{\mathrm{b}}$ Ilich A. Ibarra, ${ }^{\mathrm{c}}$ Eduardo González-Zamora, ${ }^{\mathrm{b}, *}$ Rocío Gámez-Montaño, ${ }^{\mathrm{a}, *}$ Alejandro Islas-Jácome ${ }^{\mathrm{b}, *}$

${ }^{a}$ Departamento de Química, División de Ciencias Naturales y Exactas, Universidad de Guanajuato, Noria Alta S/N, Col. Noria Alta, Guanajuato, C.P. 36050, Gto., México.

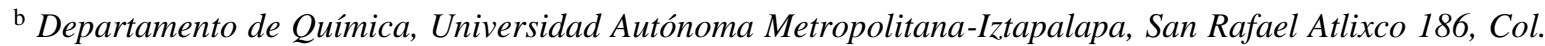
Vicentina, Del. Iztapalapa, C.P. 09340, Ciudad de México.

${ }^{\mathrm{c}}$ Instituto de Investigaciones en Materiales, Universidad Nacional Autónoma de México, Circuito Exterior S/N, CU, Del. Coyoacán, C.P. 04510, Ciudad de México.

\begin{abstract}
Eight novel 2-aminonitrile oxazoles were synthesized efficiently and quickly via an Ugi reaction in its three-component version in moderate to good yields (61-79\%) at room temperature or in good to excellent yields (73-90\%) under ultrasound irradiation (USI) conditions. It is noteworthy that not only the yields were improved by using USI, also the reaction times decreased considerably, from 3 hours (at r.t.) to 1 hour (under USI), depending on the substituents in the final products, which are highly functionalized because have an amino group, a nitrile group, an oxazole group and a very reactive methylene-linked biaryl. In this context, they can be used for further condensations, cyclizations and/or functionalizations toward a variety of compounds with potential applications in several fields of knowledge like optics, material science and medicinal chemistry.
\end{abstract}

\section{Keywords}

Ugi-3CR, oxazoles, aminonitriles, ultrasound irradiation

\section{Introduction}

The oxazole ring is an oxygen and nitrogen-containing five-membered heterocyclic system of high interest in medicinal chemistry because it is the core of various natural and synthetic products exhibiting very valuable pharmacological activities, for example, the non-steroidal anti-inflammatory drug Oxaprozin (1), ${ }^{1}$ and the antitubercular oxazole-based compound $\mathbf{2},{ }^{2}$ figure 1. Thus, we herein communicate an ultrasound-assisted multicomponent-based method toward the synthesis of the eight novel and highly functionalized 2-aminonitrile oxazoles 3a-h, which could be used for further in vitro SAR studies, as well as for further smart synthetic transformations. 


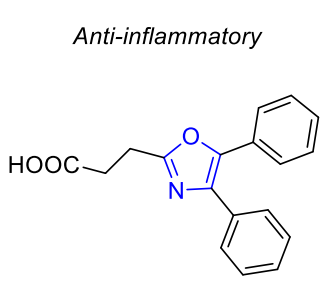

Oxaprozin (1)

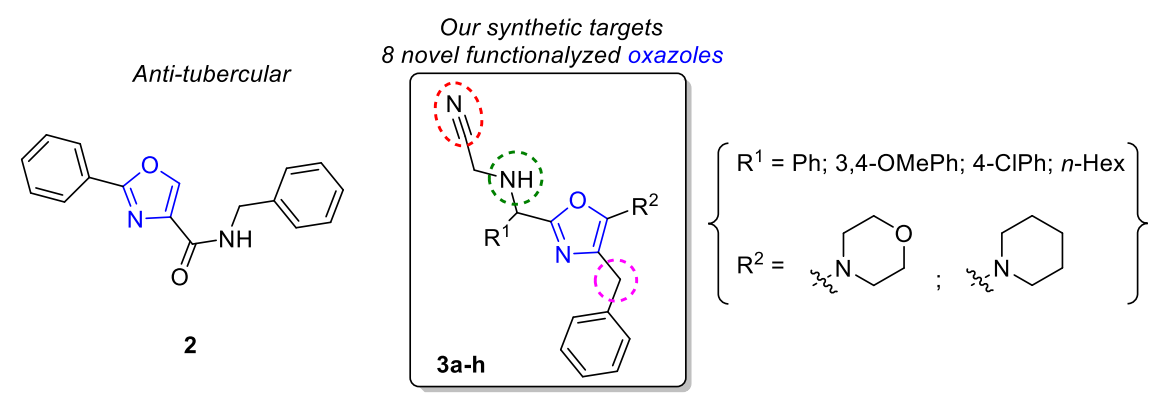

Figure 1. Selected oxazoles with pharmacological activity and our synthetic targets.

Poly-substituted oxazoles are commonly synthesized through a cyclization step involving the use of substrates prepared stepwise under harsh conditions. Thus, among all reported methods, those allowing the synthesis of highly functionalized oxazoles are via: $i$ ) visiblelight photocatalysis of $\alpha$-bromoketones with benzylamines, ${ }^{3}$ ii) intramolecular oxidative cyclization of $N$-styrylbenzamides, ${ }^{4}$ iii) $\operatorname{Copper(II)~triflate~catalyzed~coupling~of~} \alpha$ diazoketones with amides (used to synthesize analogues of Balzoxin), ${ }^{5}$ iv) $[2+2+1]$ heteroannulation of alkynes, nitriles and $O$-atoms using triflic acid (harsh acidic conditions), ${ }^{6}$ v) ring expansion of 2-carbonyl ketoaziridines (used to synthesize the Oxaprozin 1), ${ }^{7}$ and vi) via phenyl iodine diacetate (PIDA)-mediated intramolecular cyclization of enamides. ${ }^{8}$ Besides, it has been reported some multicomponent-based synthetic strategies toward a variety of polysubstituted and highly functionalized oxazoles. It is noteworthy that Multicomponent reactions (MCR's) are privileged one pot processes in which sequential combinations of at least three reagents in the same pot are involved, reducing the use of harsh conditions and the atomic waste. ${ }^{9}$ In this context, the general MCR-based method to synthesize functionalized oxazoles is the Van Leusen oxazole synthesis from aldehydes and the bifunctional TOSMIC. ${ }^{10}$ However, particularly with respect to 5 -amino oxazoles, pioneering works were performed by J. Zhu and $\mathrm{H}$. Bienaymé via a sequential combination of amines, aldehydes and ring-chain tautomerizable isocyanides (aminoacid derivatives) via the Ugi-type MCR in its three component version. ${ }^{11-13}$ Further works from J. Zhu's group were published toward 5-alcoxy oxazoles. ${ }^{14-15}$ The more plausible reaction mechanism involves a condensation of the amines $\mathbf{4}$ with the aldehydes $\mathbf{5}$ to give the intermediate imines 6, which by a further $\alpha$-nucleophilic attack of the isocyanides $\mathbf{7}$ coupled to a ring-chain tautomerizable process of the nitrilium ion 8 results in the heterocycles 3 , scheme 1.

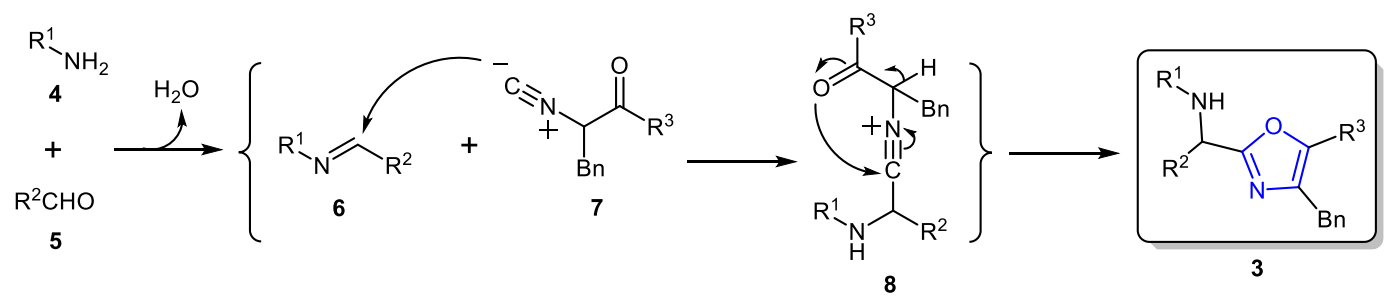

Scheme 1. Reaction mechanism involved in the Ugi-3CR toward 5-amino oxazoles 


\section{Results and discussion}

Thus, inspired on the Zhu's work and after optimizing the reaction conditions, the series of novel 5-aminonitrile oxazoles 3a-h were synthesized either, in moderate to good yields (61$79 \%)$ at room temperature or in good to excellent yields (73-90\%) under ultrasound irradiation (USI) conditions. The aminoacetonitrile (4) was combined sequentially with the aliphatic or aromatic aldehydes 5a-d (decorated with substituents having different stereoelectronic nature), and the isocyanides 6a-b (morpholine or piperidine derivatives) in anhydrous $\mathrm{MeOH}[1 \mathrm{M}]$ using scandium(III) triflate as Lewis acid catalyst to promote the $\alpha$ nucleophilic attack, scheme 2.
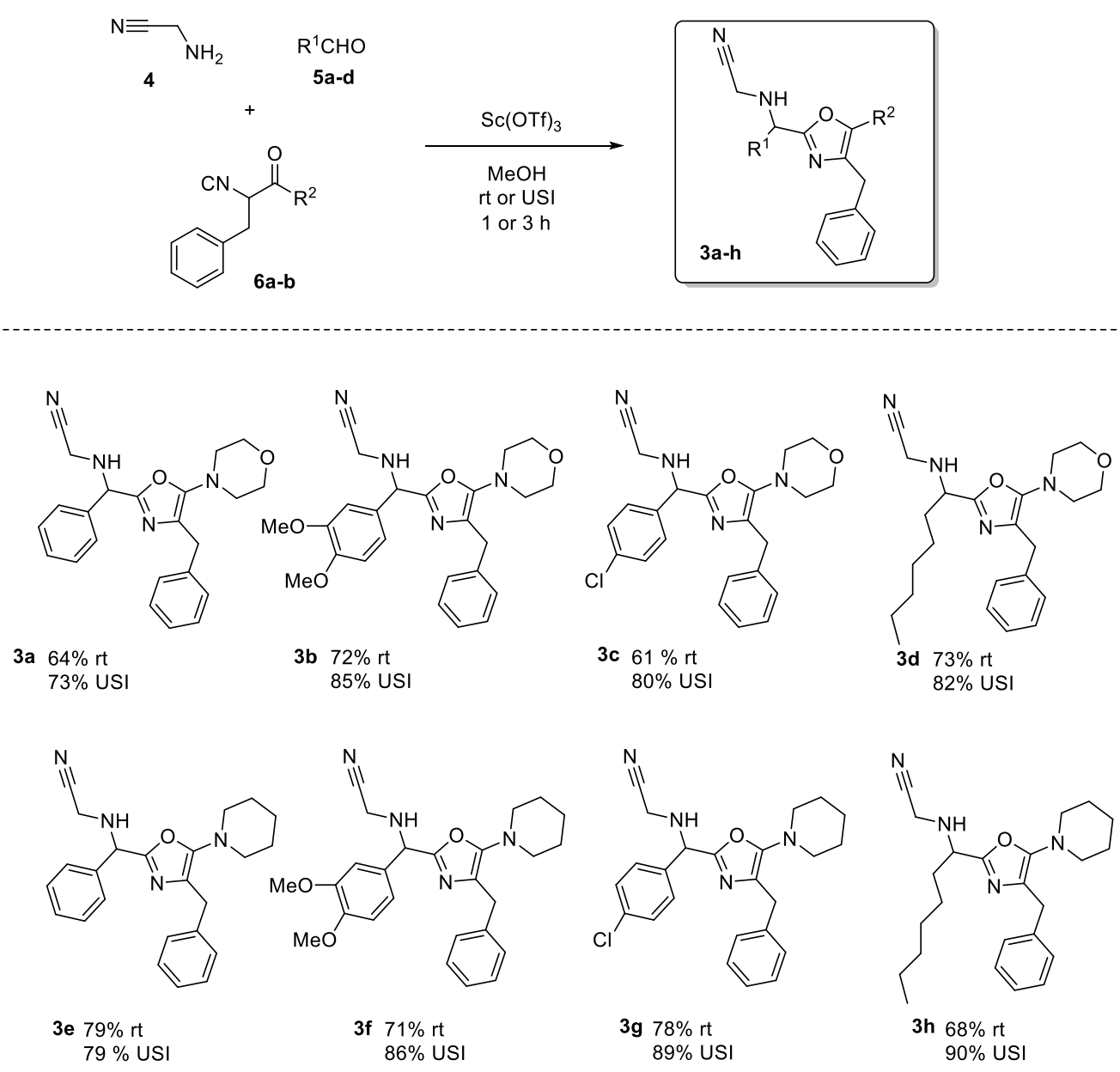

Scheme 2. Substrate scope

As seen, one more time the great usefulness of the green ultrasound irradiation (USI) was demonstrated, not only for reducing the reaction times (from 3 to $1 \mathrm{~h}$ ), but also for increasing the yields (from 61-79 to 73-90\%). It is noteworthy that no much variation in the yields were observed. This behavior may be understood in terms of the generality of this methodology. Moreover, all the synthesized products were characterized by typical spectroscopic techniques, even by $\mathrm{mp}$ and $\mathrm{R}_{f}$. 
The figure 2 shows the ${ }^{1} \mathrm{H}$ and ${ }^{13} \mathrm{C}$ NMR spectra for the selected 5-aminoacetonitrile oxazole 3a. As it can be seen, the $\mathrm{C}-\mathrm{H}$ bonding the three starting reagents (amine, aldehyde and isocyanide) appear as singlet in the H NMR spectra approximately at $5.0 \mathrm{ppm}$ and a peak in the C NMR spectra approximately at 60.0 ppm (depending on the oxazole 3a-h). All the other key signals and peaks can be found easily from these spectra,

(a)
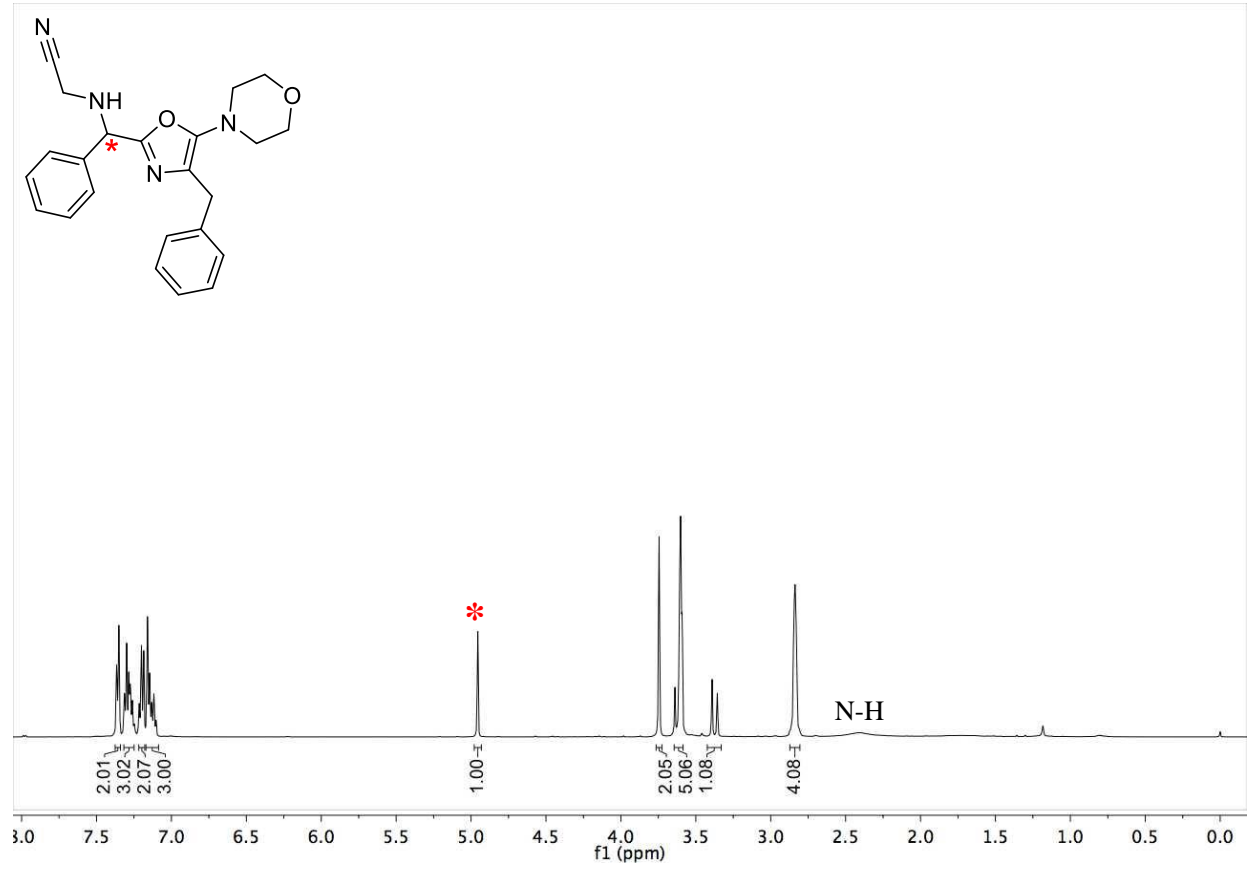

(b)
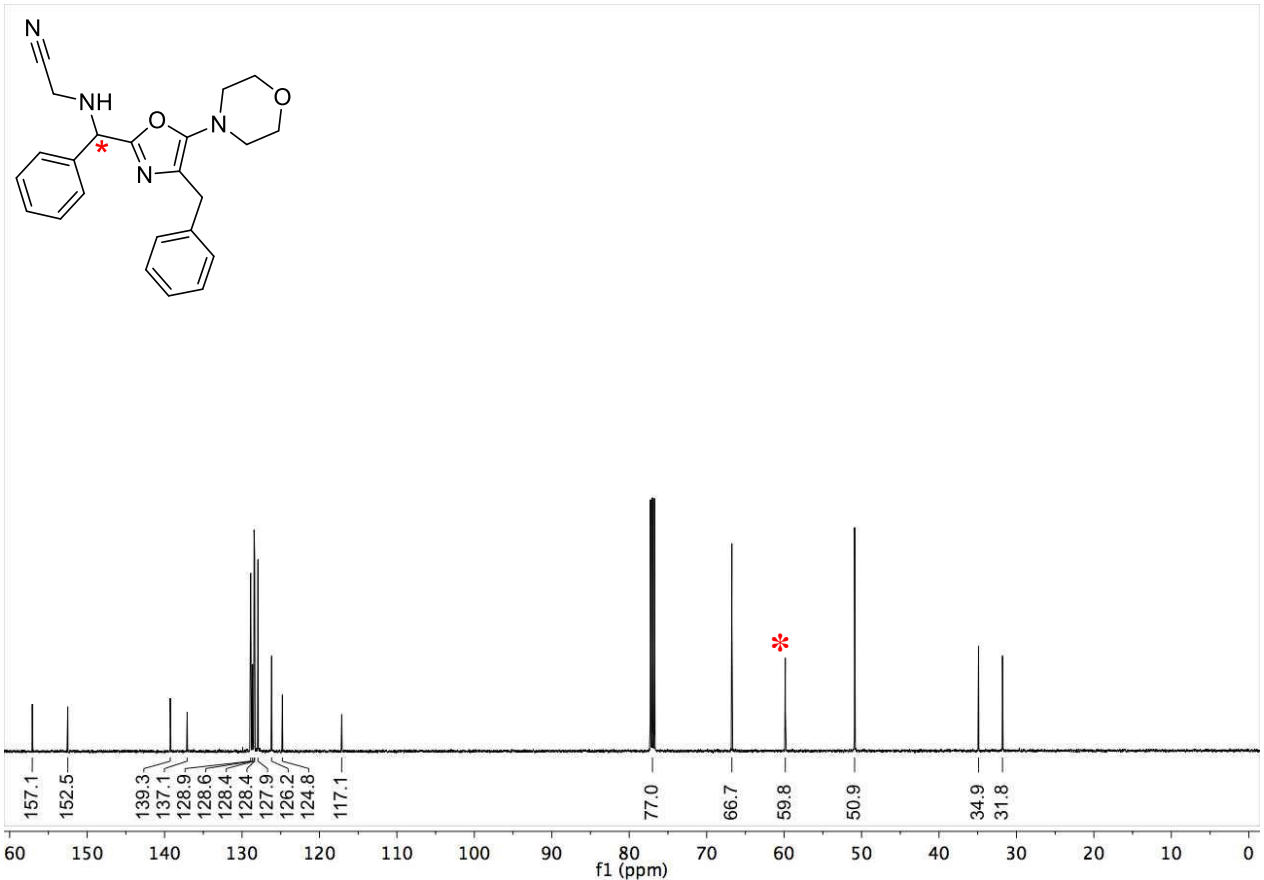

Figure 2. (a) ${ }^{1} \mathrm{H}$ and (b) ${ }^{13} \mathrm{C}$ NMR spectra for the selected 5-aminoacetonitrile oxazole $3 a$. 
The products 3a-h are highly functionalized oxazole-containing products. In this context, they can be used for further condensations, cyclizations and/or functionalizations toward a variety of compounds with potential applications in several fields of knowledge like optics, material science and medicinal chemistry. For example, the nitrile moiety can be used to perform [3+2] dipolar cyclizations. The secondary amine can be used for $N$-acyl substitutions, $\mathrm{S}_{\mathrm{N}} 2$, or for tandem-type condensations. The methylene-linked biaryl has a very short value of pKa. The oxazole ring can be used as aza-diene for Diels-Alder cycloadditions. ${ }^{16-20}$ In the same way, our compounds can be taken also as starting points for future SAR studies, for example, toward anti-inflammatories or antitubercular because there are some reports describing the usefulness of polysubstituted and polyfunctionalized oxazoles from these approaches.

\section{Acknowledgements}

I.A.I. acknowledges PAPIIT-UNAM-Mexico (IN100415) for financial support. E.G.-Z. thanks CONACyT-México (236879) for financial support. R.G.-M is grateful for financial support from the DAIP (859/2016) and CONACYT (CB-2011-166747-Q) projects. A.I.-J. acknowledges QI-DQ-CBI-UAMI for his position as visiting professor (40966) and PRODEP for financial support (12413143).

\section{Experimental part}

\section{General information, instrumentation, and chemicals}

${ }^{1} \mathrm{H}$ and ${ }^{13} \mathrm{C}$ NMR spectra were acquired on either, Bruker Advance III spectrometers (500 or $400 \mathrm{MHz})$. The solvent used was deuterated chloroform $\left(\mathrm{CDCl}_{3}\right)$. Chemical shifts are reported in parts per million $(\delta / \mathrm{ppm})$. Internal reference for ${ }^{1} \mathrm{H}$ NMR spectra is respect to TMS at $0.0 \mathrm{ppm}$. Internal reference for ${ }^{13} \mathrm{C} \mathrm{NMR}$ spectra is respect to $\mathrm{CDCl}_{3}$ at $77.0 \mathrm{ppm}$. Coupling constants are reported in Hertz $(\mathrm{J} / \mathrm{Hz})$. Multiplicities of the signals are reported using the standard abbreviations: singlet (s), doublet (d), triplet (t), quartet (q) and multiplet (m). NMR spectra were analyzed using the MestreNova software version 10.0.1-14719. IR spectra were acquired on a Perkin Elmer 100 spectrometer. The absorbance peaks are reported in reciprocal centimeters $\left(v_{\max } / \mathrm{cm}^{-1}\right)$. US-irradiated reactions were performed in sealed vials placed in a water bath of a sonicator cleaner working at frequencies of $42 \mathrm{kHz} \pm$ $6 \%$. Reaction progress was monitored by TLC on precoated Silica-gel $60 \mathrm{~F}_{254}$ plates and the spots were visualized under UV light at 254 or $365 \mathrm{~nm}$. Mixtures of hexanes (Hex) with ethyl acetate (AcOEt) were used to run TLC and for measuring retention factors $\left(\mathrm{R}_{f}\right)$. Flash column chromatography was performed using silica gel (230-400 mesh) and mixtures of Hex with AcOEt in different proportions (v/v) as mobile phase. All starting materials were purchased from Sigma-Aldrich and were used without further purification. Chemical names and drawings were obtained using the ChemBioDraw Ultra 13.0.2.3020 software package. The purity for all the synthesized products (up to 99\%) was assessed by NMR. 
Synthesis and characterization of the 6-propargyl-pyrrolo[3,4-b]pyridin-5-ones 3a-h

General procedure (GP): Aminoacetonitrile (4) (1.1 equiv.), the corresponding aldehyde 5 (1.0 equiv.) and the $\mathrm{Sc}(\mathrm{OTf})_{3}(3 \% \mathrm{~mol})$ were placed in a $10 \mathrm{~mL}$ sealed vial equipped with a magnetic stirring bar in methanol $[1.0 \mathrm{M}]$. Then, the mixture was stirred at $\mathrm{rt}$ or under USI conditions for $30 \mathrm{~min}$ and the corresponding isocyanide $\mathbf{6}$ (1.2 equiv.) was added. The new mixture was stirred at $\mathrm{rt}$ for $3 \mathrm{~h}$ or under USI conditions for $1 \mathrm{~h}$. Then, the solvent was removed to dryness under vacuum. The crude was diluted in dichloromethane $(5.0 \mathrm{~mL})$ and washed with a concentrated aq. solution of $\mathrm{NaHCO}_{3}(3 \times 15 \mathrm{~mL})$ and brine $(3 \times 15 \mathrm{~mL})$. The organic layer was dried using $\mathrm{Na}_{2} \mathrm{SO}_{4}$, filtered over celite pad and the solvent was removed to dryness under vacuum. The residue was purified via silica-gel chromatoflash using mixtures of $\mathrm{Hex}-\mathrm{EtOAc}(\mathrm{v} / \mathrm{v})$ in different proportions to afford the corresponding oxazoles 3a-h.

2-(((4-benzyl-5-morpholinooxazol-2-yl)(phenyl)methyl)amino)acetonitrile 3a

According to the GP, aminoacetonitrile $(29.0 \mathrm{mg}, 0.517 \mathrm{mmol}$ ), benzaldehyde ( $49.9 \mathrm{mg}$, $0.470 \mathrm{mmol})$, scandium(III) triflate $(7.0 \mathrm{mg}, 0.014 \mathrm{mmol})$, and the 2-isocyano-1-morpholino3-phenylpropan-1-one $(138.0 \mathrm{mg}, 0.564 \mathrm{mmol})$ were reacted together in $\mathrm{MeOH}(0.5 \mathrm{~mL})$ to afford the oxazole $3 \mathbf{a}(118.0 \mathrm{mg}, 64 \%$, rt $)$ or $\left(135.0 \mathrm{mg}, 73 \%\right.$, USI) as a white oil; $\mathrm{R}_{f}=0.31$ $(\mathrm{Hex}-\mathrm{AcOEt}=3 / 1 \mathrm{v} / \mathrm{v})$; FT-IR $(\mathrm{ATR}) v_{\max } / \mathrm{cm}^{-1} 1697(\mathrm{C}=\mathrm{O}) ;{ }^{1} \mathrm{H}$ NMR $\left(500 \mathrm{MHz}, \mathrm{CDCl}_{3}\right.$, $\left.25^{\circ} \mathrm{C}\right): \delta$ 7.38-7.34 (m, 2H), 7.32-7.25 (m, 3H), 7.22-7.18 (m, 2H), 7.17-7.09 (m, 3H), 4.96 (s, 1H), 3.75 (s, 2H), 3.65-3.58 (m, 5H), 3.37 (d, $J=17.5 \mathrm{~Hz}, 1 \mathrm{H}), 2.88-2.80$ (m, 4H), 2.39 (bs, $1 \mathrm{H}) ;{ }^{13} \mathrm{C}$ NMR $\left(126 \mathrm{MHz}, \mathrm{CDCl}_{3}, 25{ }^{\circ} \mathrm{C}\right): \delta 157.1,152.5,139.3,137.1,128.9,128.7$, $128.5,128.4,128.0,126.2,124.8,117.9,66.8,59.9,50.9,34.9,31.8$.

2-(((4-benzyl-5-morpholinooxazol-2-yl)(3,4-dimethoxyphenyl)methyl)amino)acetonitrile 3b

According to the GP, aminoacetonitrile ( $30.0 \mathrm{mg}, 0.535 \mathrm{mmol}$ ), 3,4-dimethoxybenzaldehyde ( $80.8 \mathrm{mg}, 0.486 \mathrm{mmol})$, scandium(III) triflate $(6.0 \mathrm{mg}, 0.141 \mathrm{mmol})$, and the 2-isocyano-1morpholino-3-phenylpropan-1-one (142.0 mg, $0.583 \mathrm{mmol}$ ) were reacted together in $\mathrm{MeOH}$ $(0.5 \mathrm{~mL})$ to afford the oxazole $3 \mathbf{b}(158.0 \mathrm{mg}, 72 \%, \mathrm{rt})$ or $(188.0 \mathrm{mg}, 85 \%$, USI) as a yellow oil; $\mathrm{R}_{f}=0.35(\mathrm{Hex}-\mathrm{AcOEt}=1 / 1 \mathrm{v} / \mathrm{v}) ;$ FT-IR $(\mathrm{ATR}) v_{\max } / \mathrm{cm}^{-1} 1699(\mathrm{C}=\mathrm{O}) ;{ }^{1} \mathrm{H}$ NMR $(500$ $\left.\mathrm{MHz}, \mathrm{CDCl}_{3}, 25^{\circ} \mathrm{C}\right): \delta 7.20-7.09(\mathrm{~m}, 5 \mathrm{H}), 6.90(\mathrm{~d}, J=8.3 \mathrm{~Hz}, 1 \mathrm{H}), 6.87(\mathrm{~s}, 1 \mathrm{H}), 6.76(\mathrm{~d}, J$ $=8.1 \mathrm{~Hz}, 1 \mathrm{H}), 4.90(\mathrm{~s}, 1 \mathrm{H}), 3.78(\mathrm{~s}, 3 \mathrm{H}), 3.76-3.73(\mathrm{~m}, 5 \mathrm{H}), 3.63-3.57(\mathrm{~m}, 5 \mathrm{H}), 3.35(\mathrm{~d}, J=$ $17.6 \mathrm{~Hz}, 1 \mathrm{H}), 2.87-2.81(\mathrm{~m}, 4 \mathrm{H}), 2.35(\mathrm{bs}, 1 \mathrm{H}) ;{ }^{13} \mathrm{C} \mathrm{NMR}\left(126 \mathrm{MHz}, \mathrm{CDCl}_{3}, 25^{\circ} \mathrm{C}\right): \delta 157.4$, $152.4,149.4,149.3,139.4,129.4,128.5,128.4,126.2,124.9,120.7,117.2,111.1,110.5$, $66.8,59.5,55.9,51.0,34.8,31.8$.

2-(((4-benzyl-5-morpholinooxazol-2-yl)(4-chlorophenyl)methyl)amino)acetonitrile 3c

According to the GP, aminoacetonitrile $(26.0 \mathrm{mg}, 0.463 \mathrm{mmol}), 4$-chlorobenzaldehyde $(59.2$ $\mathrm{mg}, 0.421 \mathrm{mmol})$, scandium(III) triflate $(6.0 \mathrm{mg}, 0.012 \mathrm{mmol})$, and the 2-isocyano-1morpholino-3-phenylpropan-1-one $(123.0 \mathrm{mg}, 0.505 \mathrm{mmol})$ were reacted together in $\mathrm{MeOH}$ $(0.5 \mathrm{~mL})$ to afford the oxazole $3 \mathrm{c}(109.0 \mathrm{mg}, 61 \%, \mathrm{rt})$ or $(140.0 \mathrm{mg}, 80 \%$, USI) as a withe oil; $\mathrm{R}_{f}=0.32(\mathrm{Hex}-\mathrm{AcOEt}=3 / 1 \mathrm{v} / \mathrm{v}) ;$ FT-IR $($ ATR $) v_{\max } / \mathrm{cm}^{-1} 1696(\mathrm{C}=\mathrm{O}) ;{ }^{1} \mathrm{H}$ NMR $(500$ 
$\left.\mathrm{MHz}, \mathrm{CDCl}_{3}, 25^{\circ} \mathrm{C}\right): \delta 7.29(\mathrm{~d}, J=7.5 \mathrm{~Hz}, 2 \mathrm{H}), 7.25(\mathrm{~d}, J=7.8 \mathrm{~Hz}, 2 \mathrm{H}), 7.20-7.16(\mathrm{~m}$, 2H), 7.15-7.08 (m, 3H), 4.91 (s, 1H), $3.73(\mathrm{~s}, 2 \mathrm{H}), 3.62-3.57$ (m, 5H), 3.32 (d, $J=17.6 \mathrm{~Hz}$, $1 \mathrm{H}), 2.87-2.81(\mathrm{~m}, 4 \mathrm{H}), 2.41(\mathrm{bs}, 1 \mathrm{H}) ;{ }^{13} \mathrm{C}$ NMR $\left(126 \mathrm{MHz}, \mathrm{CDCl}_{3}, 25^{\circ} \mathrm{C}\right): \delta 156.7,152.7$, 139.2, 135.7, 134.6, 129.4, 129.1, 128.5, 128.4, 126.3, 124.9, 117.1, 66.8, 59.2, 50.9, 34.9, 31.8 .

2-((1-(4-benzyl-5-morpholinooxazol-2-yl)heptyl)amino)acetonitrile 3d

According to the GP, aminoacetonitrile $(26.0 \mathrm{mg}, 0.463 \mathrm{mmol})$, heptanaldehyde $(0.060 \mathrm{~mL}$, $0.421 \mathrm{mmol})$, scandium(III) triflate $(6.0 \mathrm{mg}, 0.012 \mathrm{mmol})$, and the 2-isocyano-1-morpholino3-phenylpropan-1-one ( $123.0 \mathrm{mg}, 0.505 \mathrm{mmol})$ were reacted together in $\mathrm{MeOH}(0.5 \mathrm{~mL})$ to afford the oxazole $3 \mathbf{d}\left(123.0 \mathrm{mg}, 73 \%\right.$, rt) or $\left(138.0 \mathrm{mg}, 82 \%\right.$, USI) as a white oil; $\mathrm{R}_{f}=0.30$ $(\mathrm{Hex}-\mathrm{AcOEt}=3 / 1 \mathrm{v} / \mathrm{v}) ; \mathrm{FT}-\mathrm{IR}(\mathrm{ATR}) v_{\max } / \mathrm{cm}^{-1} 1694(\mathrm{C}=\mathrm{O}) ;{ }^{1} \mathrm{H} \mathrm{NMR}\left(500 \mathrm{MHz}, \mathrm{CDCl}_{3}\right.$, $\left.25{ }^{\circ} \mathrm{C}\right): \delta 7.22-7.10(\mathrm{~m}, 5 \mathrm{H}), 3.77-3.70(\mathrm{~m}, 3 \mathrm{H}), 3.67-3.63(\mathrm{~m}, 4 \mathrm{H}), 3.54(\mathrm{~d}, J=17.6 \mathrm{~Hz}$, $1 \mathrm{H}), 3.49(\mathrm{~d}, J=17.6 \mathrm{~Hz}, 1 \mathrm{H}), 2.96-2.87(\mathrm{~m}, 4 \mathrm{H}), 1.81-1.67(\mathrm{~m}, 3 \mathrm{H}), 1.30-1.17(\mathrm{~m}, 8 \mathrm{H})$, $0.79(\mathrm{t}, J=6.6 \mathrm{~Hz}, 3 \mathrm{H}) ;{ }^{13} \mathrm{C} \mathrm{NMR}\left(126 \mathrm{MHz}, \mathrm{CDCl}_{3}, 25^{\circ} \mathrm{C}\right): \delta 158.8,152.3,139.4,128.45$ (2), 126.2, 124.9, 117.6, 66.9, 56.3, 51.1, 35.4, 34.0, 31.8, 31.6, 28.8, 25.6, 22.5, 14.0.

2-(((4-benzyl-5-(piperidin-1-yl)oxazol-2-yl)(phenyl)methyl)amino)acetonitrile 3e

According to the GP, aminoacetonitrile $(27.0 \mathrm{mg}, 0.463 \mathrm{mmol})$, benzaldehyde $(0.043 \mathrm{~mL}$, $0.421 \mathrm{mmol})$, scandium(III) triflate $(6.0 \mathrm{mg}, 0.012 \mathrm{mmol})$, and the 2-isocyano-3-phenyl-1(piperidin-1-yl)propan-1-one $(122.0 \mathrm{mg}, 0.505 \mathrm{mmol})$ were reacted together in $\mathrm{MeOH}(0.5$ $\mathrm{mL})$ to afford the oxazole $3 \mathrm{e}(134.0 \mathrm{mg}, 79 \%$, rt) or $(134.0 \mathrm{mg}, 79 \%$, USI) as a yellow oil; $\mathrm{R}_{f}=0.30(\mathrm{Hex}-\mathrm{AcOEt}=4 / 1, \mathrm{v} / \mathrm{v})$; FT-IR $(\mathrm{ATR}) v_{\max } / \mathrm{cm}^{-1} 1658(\mathrm{C}=\mathrm{O}) ;{ }^{1} \mathrm{H}$ NMR $(500 \mathrm{MHz}$, $\left.\mathrm{CDCl}_{3}, 25{ }^{\circ} \mathrm{C}\right): \delta 7.36-7.33(\mathrm{~m}, 2 \mathrm{H}), 7.29-7.23(\mathrm{~m}, 3 \mathrm{H}), 7.19-7.14(\mathrm{~m}, 4 \mathrm{H}), 7.12-7.01(\mathrm{~m}$, $1 \mathrm{H}), 4.92(\mathrm{~s}, 1 \mathrm{H}), 3.72(\mathrm{~s}, 2 \mathrm{H}), 3.57(\mathrm{~d}, J=17.5 \mathrm{~Hz}, 1 \mathrm{H}), 3.34(\mathrm{~d}, J=17.6 \mathrm{~Hz}, 1 \mathrm{H}), 2.84$ $2.78(\mathrm{~m}, 4 \mathrm{H}), 2.35$ (bs, $1 \mathrm{H}), 1.51-1.46(\mathrm{~m}, 4 \mathrm{H}), 1.42-1.39(\mathrm{~m}, 2 \mathrm{H}) ;{ }^{13} \mathrm{C} \mathrm{NMR}(126 \mathrm{MHz}$, $\left.\mathrm{CDCl}_{3}, 25^{\circ} \mathrm{C}\right): \delta 156.5,154.0,139.7,137.4,128.6,128.5,128.4,128.0,126.1,123.4,117.3$, $66.8,59.9,52.0,34.9,31.9,25.8,23.8$.

2-(((4-benzyl-5-(piperidin-1-yl)oxazol-2-yl)(3,4-dimethoxyphenyl)methyl)amino) acetonitrile $3 \mathbf{f}$

According to the GP, aminoacetonitrile ( $27.0 \mathrm{mg}, 0.463 \mathrm{mmol}$ ), 3,4-dimethoxybenzaldehyde (73 $\mathrm{mg}, 0.421 \mathrm{mmol}$ ), scandium(III) triflate $(6.0 \mathrm{mg}, 0.012 \mathrm{mmol})$, and the 2-isocyano-3phenyl-1-(piperidin-1-yl)propan-1-one $(127.0 \mathrm{mg}, 0.505 \mathrm{mmol})$ were reacted together in $\mathrm{MeOH}(0.5 \mathrm{~mL})$ to afford the oxazole $3 \mathbf{f}(140.0 \mathrm{mg}, 71 \%$, rt $)$ or $(168.0 \mathrm{mg}, 86 \%$, USI $)$ as a yellow oil; $\mathrm{R}_{f}=0.36(\mathrm{Hex}-\mathrm{AcOEt}=1 / 1, \mathrm{v} / \mathrm{v})$; FT-IR $(\mathrm{ATR}) v_{\max } / \mathrm{cm}^{-1} 1692(\mathrm{C}=\mathrm{O}) ;{ }^{1} \mathrm{H}$ NMR $\left(500 \mathrm{MHz}, \mathrm{CDCl}_{3}, 25^{\circ} \mathrm{C}\right): \delta 7.29-7.23(\mathrm{~m}, 4 \mathrm{H}), 7.22-7.15(\mathrm{~m}, 1 \mathrm{H}), 6.99(\mathrm{~d}, J=8.0 \mathrm{~Hz}, 1 \mathrm{H})$, $6.96(\mathrm{~s}, 1 \mathrm{H}), 6.84(\mathrm{~d}, J=8.1 \mathrm{~Hz}, 1 \mathrm{H}), 4.98(\mathrm{~s}, 1 \mathrm{H}), 3.87(\mathrm{~s}, 3 \mathrm{H}), 3.83(\mathrm{~s}, 3 \mathrm{H}), 3.82(\mathrm{~s}, 2 \mathrm{H})$, 3.67 (d, $J=17.7 \mathrm{~Hz}, 1 \mathrm{H}), 3.44$ (d, $J=17.5 \mathrm{~Hz}, 1 \mathrm{H}), 2.41$ (bs, $1 \mathrm{H}), 2.94-2.88$ (m, 4H), $1.62-$ $1.56(\mathrm{~m}, 4 \mathrm{H}), 1.53-1.48(\mathrm{~m}, 2 \mathrm{H}) ;{ }^{13} \mathrm{C}$ NMR $\left(126 \mathrm{MHz}, \mathrm{CDCl}_{3}, 25^{\circ} \mathrm{C}\right): \delta 156.8,153.9,149.3$, 149.2, 139.8, 129.7, 128.5, 128.3, 126.0, 123.6, 117.3, 111.1, $110.666 .8,59.5,55.9(2), 52.0$, $34.8,31.8,25.8,23.8$. 
2-(((4-benzyl-5-(piperidin-1-yl)oxazol-2-yl)(4-chlorophenyl)methyl)amino)acetonitrile 3g

According to the GP, aminoacetonitrile $(26.0 \mathrm{mg}, 0.463 \mathrm{mmol})$, 4-chlorobenzaldehyde (59.0 $\mathrm{mg}, 0.421 \mathrm{mmol})$, scandium(III) triflate $(6.0 \mathrm{mg}, 0.013 \mathrm{mmol})$, and the 2-isocyano-3-phenyl1-(piperidin-1-yl)propan-1-one $(122.0 \mathrm{mg}, 0.505 \mathrm{mmol})$ were reacted together in $\mathrm{MeOH}(0.5$ $\mathrm{mL})$ to afford the oxazole $3 \mathrm{~g}(139.0 \mathrm{mg}, 78 \%, \mathrm{rt})$ or $(158.0 \mathrm{mg}, 89 \%$, USI) as a yellow oil; $\mathrm{R}_{f}=0.33(\mathrm{Hex}-\mathrm{AcOEt}=3 / 1, \mathrm{v} / \mathrm{v}) ; \mathrm{FT}-\mathrm{IR}(\mathrm{ATR}) v_{\max } / \mathrm{cm}^{-1} 1696(\mathrm{C}=\mathrm{O}) ;{ }^{1} \mathrm{H}$ NMR $(500 \mathrm{MHz}$, $\left.\mathrm{CDCl}_{3}, 2{ }^{\circ} \mathrm{C}\right): \delta 7.29(\mathrm{~d}, J=7.9 \mathrm{~Hz}, 2 \mathrm{H}), 7.25(\mathrm{~d}, J=7.8 \mathrm{~Hz}, 2 \mathrm{H}), 7.20-7.17(\mathrm{~m}, 2 \mathrm{H}), 7.16-$ $7.13(\mathrm{~m}, 2 \mathrm{H}), 7.12-7.08(\mathrm{~m}, 1 \mathrm{H}), 4.90(\mathrm{~s}, 1 \mathrm{H}), 3.71(\mathrm{~s}, 2 \mathrm{H}), 3.58(\mathrm{~d}, J=17.5 \mathrm{~Hz}, 1 \mathrm{H}), 3.32$ $(\mathrm{d}, J=17.3 \mathrm{~Hz}, 1 \mathrm{H}), 2.87-2.76(\mathrm{~m}, 4 \mathrm{H}), 2.38$ (bs, 1H), 1.50-1.46 (m, 4H), 1.43-1.38 (m, $2 \mathrm{H}) ;{ }^{13} \mathrm{C} \mathrm{NMR}\left(126 \mathrm{MHz}, \mathrm{CDCl}_{3}, 25{ }^{\circ} \mathrm{C}\right): \delta 156.0,154.1,139.6,134.5,129.4,129.1,128.5$, $128.4,126.1,123.5,117.1,59.2,52.0,34.9,31.9,25.8,23.8$.

2-((1-(4-benzyl-5-(piperidin-1-yl)oxazol-2-yl)heptyl)amino)acetonitrile $\mathbf{3 h}$

According to the GP, aminoacetonitrile $(26.0 \mathrm{mg}, 0.463 \mathrm{mmol})$, heptanaldehyde $(0.060 \mathrm{~mL}$, $0.421 \mathrm{mmol})$, scandium(III) triflate $(6.0 \mathrm{mg}, 0.013 \mathrm{mmol})$, and the 2-isocyano-3-phenyl-1(piperidin-1-yl)propan-1-one $(122.0 \mathrm{mg}, 0.505 \mathrm{mmol})$ were reacted together in $\mathrm{MeOH}(0.5$ $\mathrm{mL})$ to afford the oxazole $3 \mathrm{~h}(113.0 \mathrm{mg}, 68 \%, \mathrm{rt})$ or $(150.0 \mathrm{mg}, 90 \%$, USI) as a yellow oil; $\mathrm{R}_{f}=0.30(\mathrm{Hex}-\mathrm{AcOEt}=4 / 1, \mathrm{v} / \mathrm{v}) ; \mathrm{FT}-\mathrm{IR}(\mathrm{ATR}) v_{\max } / \mathrm{cm}^{-1} 1699(\mathrm{C}=\mathrm{O}) ;{ }^{1} \mathrm{H} \mathrm{NMR}(400 \mathrm{MHz}$, $\left.\mathrm{CDCl}_{3}, 25{ }^{\circ} \mathrm{C}\right): 7.29-7.09(\mathrm{~m}, 5 \mathrm{H}), 4.49(\mathrm{t}, J=7.1 \mathrm{~Hz}, 1 \mathrm{H}), 3.74-3.69(\mathrm{~m}, 1 \mathrm{H}), 3.59-3.53$ (m, 1H), 3.50 (s, 1H), 3.46-3.40 (m, 1H), 3.37-3.31 (m, 1H), 3.23-3.15 (m, 2H), 3.13-3.06 $(\mathrm{m}, 1 \mathrm{H}), 2.90-2.86(\mathrm{~m}, 2 \mathrm{H}), 1.79-1.62(\mathrm{~m}, 2 \mathrm{H}), 1.60-1.41(\mathrm{~m}, 8 \mathrm{H}), 1.33-1.13(\mathrm{~m}, 6 \mathrm{H}), 0.84-$ $0.77(\mathrm{~m}, 2 \mathrm{H}) ;{ }^{13} \mathrm{C} \mathrm{NMR}\left(101 \mathrm{MHz}, \mathrm{CDCl}_{3}, 25{ }^{\circ} \mathrm{C}\right): \delta 166.8,161.6,159.3,149.1,139.7$, 128.9, 128.1, 123.9, 123.1, 77.9, 72.1, 60.2, 54.2, 39.7, 31.5, 29.6, 29.3, 29.1, 26.3, 23.9, $22.6,22.5,14.0$.

The authors declare no competing financial interests. Samples of the products 3a-h are available from the authors.

\section{References}

1.- Fernandes, E.; Costa, D.; Toste, S.A.; Lima, J.L.F.C.; Reis, L. Free Rad. Biol. Med. 2004, $37,1895$.

2.- Moraski, G.C.; Markley, L.D.; Chang, M.; Cho, S.; Franzblau, S.G.; Hwang, C.H.; Boshoff, H.; Miller, M.J. Bioorg. Med. Chem. 2012, 20, 2214.

3.- Chatterjee, T.; Cho, J.Y.; Cho, E.J. J. Org. Chem. 2016, 81, 6995.

4.- Hempel, C.; Nachtsheim, B.J. Synlett 2013, 24, 2119.

5.- Reddy, M.R.; Reddy, G.N.; Mehmood, U.; Hussein, I.A.; Rahman, S.U.; Harrabi, K.; Reddy, B.V.S. Synthesis 2015, 47, 3315.

6.- Saito, A.; Taniguchi, A.; Kambara, Y.; Hanzawa, Y. Org. Lett. 2013, 15, 2672. 
7.- Samimi, H.A.; Mohammadi, S. Synlett 2013, 24, 223.

8.- Zheng, Y.; Li, X.; Ren, C.; Zhang-Negrerie, D.; Du, Y.; Zhao, K. J. Org. Chem. 2012, $77,10353$.

9.- Ramón D.J.; Yus, M. Angew. Chem. Int. Ed. 2005, 44, 1602.

10.- Van Leusen, A. Tetrahedron Lett. 1972, 13, 3114.

11.- Sun, X.; Janvier, P.; Zhao, G.; Bienaymé, H.; Zhu, J. Org. Lett. 2001, 3, 877.

12.- Janvier, P.; Sun, X.; Bienaymé, H.; Zhu, J. Am. Chem. Soc. 2002, 124, 2560.

13.- Bughin, C.; Zhao, G.; Bienaymé, H.; Zhu, J. Chem. Eur. J. 2006, 12, 1174.

14.- Cuny, G.; Gámez-Montaño, R.; Zhu, J. Tetrahedron 2004, 60, 4879

15.- Bonne, D.; Dekhane, M.; Zhu, J. Angew. Chem. Int. Ed. 2007, 46, 2485.

16.- Islas-Jácome, A.; González-Zamora, E.; Gámez-Montaño, R. Tetrahedron Lett. 2011, 52,5245 .

17.- Islas-Jácome, A.; Cárdenas-Galindo, L. E.; Jerezano, A. V.; Tamariz, J.; GonzálezZamora, E.; Gámez-Montaño, R. Synlett 2012, 23, 2951.

18.- Islas-Jácome, A.; Gutierrez-Carrillo, A.; García-Garibay, M. A.; González-Zamora, E. Synlett 2014, 25, 403.

19.- Islas-Jácome, A.; Rentería-Gómez, A.; Rentería-Gómez, M. A.; González-Zamora, E.; Jiménez-Halla, J.O.C.; Gámez-Montaño, R. Tetrahedron Lett. 2016, 57, 3496.

20.- Vázquez-Vera, O.; Sánchez-Badillo, J.S.; Islas-Jácome, A. Rentería-Gómez, M.A.; Pharande, S.G.; Cortes-García, C.J.; Rincón-Guevara, M.A.; Ibarra, I.A.; Gámez-Montaño, R.; González-Zamora, E. Org. Biomol. Chem. 2017, 15, 2363. 